\title{
VIVÊNCIAS SOBRE O PROCESSO DE TRABALHO DAS EQUIPES DE SAÚDE DA FAMÍLIA DO MUNICÍPIO DE GURUPI- TO
}

\author{
Experience about the work process of the Health Teams of the Gurupi-TO municipal \\ Family
}

Vivencias sobre el processo de trabajo de los Equipos de Salud de la Familia GurupiMunicipal

\section{Jeann Bruno Ferreira da Silva*1, Thaís Valadão Reis ${ }^{2}$, Hericácia de Sousa Rocha ${ }^{3}$ \\ ${ }^{1}$ Psicólogo, Mestre em Ciências da Saúde pela Universidade Federal do Tocantins, Docente na Universidade de Gurupi, Tocantins, Brasil.}

${ }^{2}$ Psicóloga Clínica, Especialista em Psicologia Hospitalar pela Faculdade de Ciências Sociais Aplicadas de Marabá, Uruaçu, Goiás, Brasil.

${ }^{3}$ Psicóloga Clínica, Monte Alegre, Goiás, Brasil.

*Correspondência: Departamento de Psicologia da Universidade de Gurupi-UnirG, Av. Rio de Janeiro $n^{\circ} 1585$, Centro, Gurupi, Tocantins, Brasil. CEP:77.403-090. E-mail: jbpsicologia@live.com.

\section{Artigo recebido em 25/02/2019 aprovado em 07/10/2019 publicado em --/--/----.}

\section{RESUMO}

Dentre os desafios diários presentes no processo de trabalho dos profissionais da Atenção Básica, destacam-se as vivências de situações referentes à qualidade no atendimento aos usuários e articulação de saberes com os demais profissionais da saúde em prol do bem estar dos pacientes. Objetivou-se compreender a percepção dos coordenadores das Equipes de Saúde da Família/ESF de Gurupi-TO sobre seu processo de trabalho. Trata-se de um estudo qualitativo, de caráter exploratório-descritivo. A amostra foi composta por 10 enfermeiros coordenadores das ESF no município de Gurupi-TO. Os dados foram coletados em único momento no mês de outubro de 2017 com um coordenador de cada UBS por meio de roteiro de entrevista semiestruturada, analisados e categorizados segundo a análise de conteúdo de Bardin. Os resultados apontaram que 50\% dos enfermeiros não se encontram satisfeitos com a estrutura física das UBS que trabalham. Concluiu-se que a maioria dos profissionais se encontra descontente com as condições oferecidas no seu processo de trabalho, como a estrutura física inadequada à demanda, déficit de profissionais, excesso de atribuições e ausência de veículos oficiais para locomoção da zona urbana para a rural.

Palavras-chave: Processo de trabalho; Profissionais da Saúde; Atenção Básica.

\begin{abstract}
Among the daily challenges of the work process of the professionals of primary care, are the experiences of situations that require quality in the care of users and articulation of knowledge with other health professionals in favor of the welfare of patients. The objective was to understand the perceptions of the coordinators of the Family health teams/FHS of Gurupi-TO on their work process. This is a qualitative, exploratory-descriptive study. The sample consisted of 10 nurse coordinators of the FHS in the municipality of Gurupi-TO. Data were collected in a single moment in the month of October 2017 mounth with the coordinator of each UBS by means of a semi-structured interview script, analyzed and categorized according to Bardin's content analysis. The results showed that 50\% of the nurses are not satisfied with the physical structure of the UBS that work. It was concluded that most professionals are unhappy with the conditions offered in their work process, such as inadequate physical structure to demand, deficit of professionals, excessive attributions and absence of official vehicles for Urban-rural locomotion.
\end{abstract}

Keywords: work process; Health professionals; Basic attention. 


\section{RESUMEN}

Entre los retos cotidianos del proceso de trabajo de los profesionales de la atención primaria, se encuentran las experiencias de situaciones que requieren calidad en el cuidado de los usuarios y la articulación del conocimiento con otros profesionales de la salud en favor del bienestar de los pacientes. El objetivo era entender las percepciones de los coordinadores de los equipos de salud de la familia/ESF de Gurupi-TO en su proceso de trabajo. Se trata de un estudio cualitativo, exploratorio-descriptivo. La muestra consistió en 10 coordinadores de la enfermera del ESF en el municipio de Gurupi-TO. Los datos fueron recogidos en un solo momento en el mes de octubre 2017 con el Coordinador de cada UBS por medio de un guión de entrevista semiestructurado, analizado y categorizado según el análisis de contenido de Bardin. Los resultados mostraron que el $50 \%$ de las enfermeras no están satisfechos con la estructura física de los UBS que trabajan. Se concluyó que la mayoría de los profesionales no están satisfechos con las condiciones ofrecidas en su proceso de trabajo, como la inadecuada estructura física de la demanda, el déficit de profesionales, las atribuciones excesivas y la ausencia de vehículos oficiales para Locomoción urbanorural.

Palabras clave: proceso de trabajo; Profesionales de la salud; Atención básica.

\section{INTRODUÇÃO}

Atualmente, o trabalho é concebido como uma atividade que envolve o homem em todas suas dimensões, exercendo importante papel na construção da subjetividade humana, e como tal, um elemento constitutivo da saúde mental e coletiva (ANCHIETA, ANCHIETA; GALINKIN; MENDES, NEIVA; 2011).

Dentre os desafios diários do processo de trabalho dos profissionais da Atenção Básica estão as vivências de situações que exigem de si qualidade no atendimento aos usuários, bem como articulação de seus saberes com demais profissionais de diferentes formações em prol de objetivos comuns.

Sob esta perspectiva, é necessário discutir acerca do real do trabalho, o qual se apresenta ao sujeito por meio de um efeito surpresa desagradável, ou seja, de maneira afetiva. É sempre afetivamente que o real do mundo se manifesta para o sujeito, mas ao mesmo momento que o sujeito experimenta essa afetividade, também sente o sofrimento no próprio corpo (DEJOURS, 2004).

Diante ao exposto, é necessário compreender as estratégias mecanismos defensivos estruturados pelos trabalhadores, os quais vivenciam em sua práxis uma luta constante, transitando entre o prazer e o sofrimento, de acordo com as demandas que lhes são conferidas no cotidiano.

Atualmente as equipes de Atenção Básica (eAB) têm, na sua atuação, a UBS como local prioritário para sua práxis, desenvolvendo a Atenção Básica ( $\mathrm{AB}$ ) com elevado grau de descentralização e profunda capilaridade no território nacional, com vistas na aproximação do cotidiano das pessoas (BRASIL, 2012). Essa atuação requer tamanho desdobramento do profissional sobre seu fazer em saúde.

Partindo ao encontro de identificar a percepção que o trabalhador tem sobre seu fazer, a Política Nacional de Promoção Saúde (PNPS) vem apresentando, na última década, a importância do cuidado sobre a vida, abrangendo a vulnerabilidade do adoecimento, e ainda, mostrando que os processos de adoecimento são resultantes da organização da produção do trabalho na sociedade (BRASIL, 2010).

Este trabalho surgiu a partir do interesse em pesquisar sobre as representações que o processo de trabalho pode proporcionar aos profissionais do campo da saúde, especificamente aqueles que atuam na Atenção Básica (AB). Assim, cabe refletir sobre as possibilidades de articular sua atuação dentro das 
perspectivas multi, inter e transdisciplinar por meio dos elementos já existentes na realidade cotidiana.

A equipe de Saúde da Família (eSF) se encontra num processo de trabalho direcionado por duas vertentes, uma é a gestão, que exige efetivação de metas e produção, e a outra centra-se na figura do usuário do Sistema Único de Saúde (SUS), que exigem melhores condições mais humanas nos cuidados oferecidos pelas eAB (BRASIL, 2012).

Os ambientes de trabalho em saúde estão estruturados de tal forma que não contribuem para o cuidado dos cuidadores, contrariamente, são meios onde existe esgotamento físico e mental, frente a uma dicotomia entre o cuidar do outro e não cuidar de si (DE ARAUJO FREITAS, 2015).

Por essa rotina estressante, os profissionais de saúde estão sujeitos a se depararem com situações que instabilizam o relacionamento interpessoal com os demais colegas de equipe e comprometerem diretamente o atendimento aos usuários do SUS (HOGA, 1998).

Em consonância ao exposto, é relevante considerar o profissional como principal sujeito no ambiente de trabalho, dessa forma, é importante identificar a percepção do coordenador das ESF no seu processo de trabalho, como o processo de trabalho é organizado na UBS e os aspectos do cotidiano que influenciam no ambiente de trabalho.

Dessa forma, considera-se que os coordenadores das ESF compreendem os impasses do processo de trabalho na Atenção Básica, e zelam para manutenção do bem estar em suas rotinas, sendo o trabalho em equipe o principal meio para tal finalidade.

Diante ao exposto, o trabalho teve por objetivo primário compreender a percepção dos coordenadores das equipes de saúde da família de Gurupi- TO sobre o seu processo de trabalho. Os objetivos secundários desdobraram-se em caracterizar o perfil sociodemográfico dos coordenadores, conhecer a organização e os aspectos do cotidiano do serviço das Equipes de Saúde da Família.

\section{MATERIAIS E MÉTODOS}

Trata-se de um estudo qualitativo, de caráter exploratório-descritivo. A amostra foi recrutada nas Equipes de Saúde da Família atuantes no município de Gurupi, interior do estado do Tocantins. Das 11 unidades básicas de saúde foram entrevistados 10 enfermeiros coordenadores das ESF no mês de outubro do ano de 2017, sendo 1 de sua respectiva lotação, que estavam na unidade no período mínimo de seis meses e aceitaram participar da pesquisa assinando o Termo de Consentimento Livre e Esclarecido (TCLE). Excluíram-se da amostra sujeitos que não coordenassem as Equipes de Saúde da Família, profissionais que exerçam funções de teor administrativo, aqueles que se encontravam de férias ou licença de qualquer natureza e os demais membros da Equipe de Saúde que não aceitaram participar do estudo, recusando-se a assinar o TCLE.

O estudo ocorreu conforme previsto na resolução 466/2012 do Conselho Nacional de Saúde, foi submetido ao Comitê de Ética em Pesquisa com Seres Humanos sob CAAE: 73315717.0.0000.5518 e aprovado conforme parecer $\mathrm{n}^{\circ} 2.240 .713$.

Os dados foram coletados em único momento com o coordenador de cada UBS por meio de roteiro de entrevista semiestruturada. Também foi utilizado diário de campo com o propósito de observar e registrar a dinâmica do local, percepções e sentimentos do pesquisador acerca de tal contexto.

Em cada UBS, a coleta ocorreu nas salas de atendimento de enfermagem de maneira individual. Todas as informações obtidas foram gravadas e transcritas na íntegra para posterior análise dos dados. Cada entrevista teve duração de 40 (quarenta) minutos. 
Os dados foram analisados e categorizados segundo a análise de conteúdo de Bardin (2011), a partir de uma pré-análise (organizando o material coletado e sistematizando as ideias através de leitura meticulosa das respostas obtidas) e, a seguir, realizada a categorização das unidades de registro. $\mathrm{Na}$ fase da exploração do material a análise exigiu sua codificação, ou seja, sua transformação de dados brutos dos textos por recortes, agregação ou enumeração, até que sua codificação atingiu a representação do conteúdo ou sua expressão.

A última etapa consistiu em codificar o material, incluindo a escolha de unidades de registro e a seleção de regras de contagem. A unidade de registro (UR) é a unidade de significação a codificar, a qual pode ser o tema, palavra ou frase, sendo que a frequência com que aparece uma Unidade de Registro denota-lhe importância, a Unidade de Contexto (UC) representa a categoria macro das UR, as Categorias Finais de Análise representam o tratamento final das UC (BARDIN, 2011).

\section{RESULTADOS E DISCUSSÃO}

As UBS são configuradas como ambientes em que existem frequentes tensões, nos quais os trabalhadores enfrentam diversas situações e fatores que afetam a sua integridade física, psíquica e emocional, visto que é dever da equipe de saúde acolher a população, atentando-se para suas inúmeras necessidades e demandas (DE ARAUJO FREITAS, 2015).

Ao serem questionados sobre sugestões para o pleno desenvolvimento do trabalho na UBS, os entrevistados E1 e E8 relataram:

"[...] Eu acho que deveria assim, já que é só uma vez na semana, eu acho assim, que deveria ser feito uma, um planejamento já pra destinar um veículo, já pra todo esse dia da semana que a equipe vai lá atender a população já tá pronto, sabe! Isso vez ou outra gera muito desconforto, que a gente se programa, se planeja [...]”. (E1)

"[...] a gente está precisando muito era outra equipe, porque aqui a população ela é muito, já cresceu muito e nós já estamos assim superlotado tanto que nós não consegue suprir a demanda que tem, porque aqui a população é bem carente". (E8).

Outro fato a ser pontuado refere-se à humanização nos serviços de saúde, que reúne estratégias para eficácia e eficiência no atendimento ao usuário e nas relações de trabalho. A humanização tornou-se uma preocupação dos profissionais de saúde, funcionários e gestores, representando um fator a ser considerado para se ter excelência na qualidade do atendimento em saúde, principalmente em relação aos idosos, devido às condições especiais que apresentam (LIMA, 2010).

Sobre o processo de humanização nos serviços de saúde, os entrevistados E2, E3 e E8 apresentaram os seguintes relatos:

“[...] dando atenção a cada paciente de acordo com cada problema, com cada problemática, né que ele tráz, tratando cada paciente como um ai eu acredito que essa visão da enfermagem ela é bastante importante na equipe". (E2)

“[...] o atendimento também ao paciente que a gente atende bastante os pacientes, um acolhimento que a gente faz, uma classificação de risco, observar o que o paciente realmente precisa, conseguir orientar bem o paciente, ver a questão do fluxograma de encaminhamento que ele tem, os retornos que ele tem que fazer, acompanhar, ter um 
acompanhamento, que o postinho é isso acompanhar o paciente, é algo continuo né, não é um atendimento que para, mas ele é continuo". (E3)

"[...] Pelas dificuldades que a gente tem a gente tenta prestar um melhor atendimento que a gente pode né, nas nossas limitações a gente sempre tenta fazer ao máximo pelo nosso paciente, então é bom mesmo". (E8)

A relação profissional-equipe-usuário é marcada por vivências que envolvem acolhimento, empatia e cumprimento de normas, exigindo esforço técnico e emocional do trabalhador em saúde, que diante das diversas situações inerentes à sua práxis, põe em primeiro plano a eficiência e qualidade do serviço, podendo repercutir consideravelmente em sua saúde mental.

Neumann (2007, p. 35), acrescenta que o acolhimento expresso revela a intersubjetividade necessária para suportar situações de estresse no trabalho. O ser humano tem a necessidade de ser cuidado e acarinhado. Os enfermeiros, ao serem questionados sobre o vínculo com sua respectiva equipe que coordena, os mesmos relataram ser satisfatório.

"[...] aqui nessa unidade de saúde a equipe ela é bem unida, bem participativa, compreensiva, a gente tem um bom relacionamento, tanto aqui dentro dos profissionais, como nos outro no ambiente fora a secretaria de saúde e as outras parcerias que a gente faz também [...].” (E5)

“[...] tanto de trabalho como de convivência também em equipe não tenho o que reclamar não, muito boa, são todo mundo assim bem, não tem ninguém assim problemático assim, não tem nenhum problema entre equipe, não tem nada não, todo mundo se dá bem, todo mundo se dá bem aqui na verdade [...].” (E6)

Outro fato identificado nas entrevistas referese à estrutura física das UBS, que segundo a percepção dos entrevistados, as mesmas não possuem a estrutura física adequada à demanda. Este aspecto é relatado nas entrevistas abaixo:

"[...] eu avalio que tem pouca sala pra quantidade de atendimentos que nós temos que fazer né?! (E2)

“[...] então ele não foi feito dentro de um planejamento de unidade básica de saúde, como tem que ser feito, como dentro das normas, dentro das diretrizes, então ela não é uma estrutura boa, isso não interfere no trabalho, a gente trabalha bem dentro do que tem, mas não é correto de acordo com a estrutura física que teria que ser. (E3)

"A nossa estrutura física como vocês mesmo estão vendo que ela é bem precária, assim a gente tem muita dificuldade em questão de estrutura física porque que nós adaptamos muita coisa, então por exemplo, na sala da triagem lá fica a triagem, sala de curativo, sala de vacina, tudo muito assim muito cheio as salas, aqui na minha sala é minha sala e a sala de citologia que não tem nenhum banheiro, sabe?! [...].” (E8)

Esse dado que vem a corroborar com a pesquisa realizada por Teixeira (2007) pontuando que diversos fatores no meio ambiente de trabalho podem afetar a saúde mental dos profissionais da saúde, tais como: relações coletivas inerentes a organização do trabalho, o ambiente físico, por exemplo, ruídos, oscilação de temperatura, intoxicação etc.

Para conseguir desempenhar um trabalho com eficiência nas UBS, seja na gestão ou na assistência, o 
enfermeiro necessita ter à sua disposição meios de trabalho, principalmente os consultórios e ambientes de recepção, além de instrumentos essenciais para a realização de diagnostico, vacinas e medicamentos, e tudo que for fundamental no atendimento ao usuário de maneira satisfatória em saúde.

Contudo, os entrevistados E4 e E7 avaliam de forma positiva a estrutura física de suas respectivas UBS.

"Muito bom, ótima. Aqui os ambientes é tudo climatizado o posto é grande, tem sala pra atender todo mundo, banheiro em todo os consultórios, então considero muito bom”. (E4)

“[...] Como você pode ver todas as salas, tem sala, banheiro, o espaço é bom, tem a sala de reunião, as salas são espaçosas, tem sala de fisioterapia. Eu gosto, gosto bastante [...]." (E7)

É de responsabilidade do governo garantir a infraestrutura necessária ao funcionamento das Unidades Básicas de Saúde. Compete as Secretarias Municipais da Saúde garantir a estrutura física necessária para o funcionamento das UBS e para a execução do conjunto de ações propostas, podendo contar com apoio técnico e/ou financeiro das Secretarias de Estado da Saúde e do Ministério da Saúde. E ainda responsabilizar-se de recursos materiais e equipamentos suficientes para o funcionamento das UBS (BRASIL, 2012).

É indispensável que para o pleno funcionamento das UBS, o Ministério da Saúde recomenda-se, consultório médico/enfermagem; consultório odontológico e consultório com sanitário; sala multiprofissional de acolhimento à demanda espontânea; sala de administração e gerência; e sala de atividades coletivas para os profissionais da atenção básica; área de recepção, local para arquivos e registros; sala de procedimentos; sala de vacinas; área de dispensação de medicamentos e sala de armazenagem de medicamentos (quando há dispensação na UBS); sala de inalação coletiva; sala de procedimentos; sala de coleta; sala de curativos; sala de observação, entre outros (BRASIL, 2012).

Salienta-se também que existem características estruturais a serem observadas. A estrutura das Unidades Básicas de Saúde deve enfocar as instalações elétricas e hidráulicas, ventilação, luminosidade, fluxo de usuários e facilidade na limpeza e desinfecção.

A ventilação adequada é indispensável para se manter a salubridade das UBS. Recomenda-se que todos os ambientes sejam claros, com o maior iluminação natural possível. Todo o projeto da estrutura física da UBS deve permitir o acesso de pessoas deficientes e de pessoas com limitações. Deve-se restringir o acesso de algumas pessoas aos ambientes, com o objetivo de se conseguir maior controle de transmissão de infecção, dentre outras (BRASIL, 2008).

\section{CONCLUSÃO}

Sobre a percepção dos Coordenadores das ESF de Gurupi em relação ao seu processo de trabalho, inferiu-se que a maioria se encontra descontente com as condições oferecidas. Este fato pode ser evidenciado a partir da identificação de variáveis, tais como a estrutura física inadequada à demanda, déficit de profissionais, excesso de atribuições para o enfermeiro e ausência de veículos oficiais para locomoção da zona urbana para a rural.

Entretanto, mesmo diante de tais dificuldades, prevalece o bom relacionamento dos colaboradores entre si. Infere-se que este seja um fator positivo para um pleno desenvolvimento do processo de trabalho dentro das UBS. 
Pesquisar sobre a Atenção Básica foi uma proposta desafiadora, contudo, no decorrer das etapas, foi possível conhecer as diferentes perspectivas, vivências e sentimentos sobre o significado do trabalho para cada entrevistado.

Não se pretende esgotar a discussão a respeito do tema em questão. De tal modo, pretendemos com este estudo contribuir para a construção do conhecimento sobre o tema e para reflexões sobre os sentimentos e vivências dos enfermeiros das UBS.

Sugere-se que sejam realizados novos estudos voltados para os enfermeiros, estudos estes com base na avaliação da real situação de saúde dos mesmos e que possam contribuir para a investigação da sua saúde física e mental, com implementações de ações estratégicas favoráveis à saúde dos mesmos.

\section{AGRADECIMENTOS}

Agradecemos todos os envolvidos nesse estudo, especificamente aos enfermeiros que acolheram os pesquisadores em meio à suas respectivas jornadas de trabalho. Oferecemos-lhes nossa gratidão.

Todos os autores declararam não haver qualquer potencial conflito de interesses referente a este artigo.

\section{REFERÊNCIAS}

ANCHIETA, Vânia Cristine Cavalcante. GALINKIN, Ana Lúcia. MENDES, Ana Magnólia Bezerra. NEIVA, Elaine Rabelo. Trabalho e riscos de adoecimento: um estudo entre policiais civis. Psicologia: Teoria e Pesquisa, v. 27, n. 2, p. 199-208, $2011 . \quad$ Disponivel em: <http://www.scielo.br/scielo.php?script=sci_arttext\& pid=S0102-37722011000200007 > Acesso em: 20 de Março de 2017.

BARDIN, Laurence. Análise de Conteúdo/Laurence Bardin; Tradução Luís Antero Reto, Augusto Pinheiro. São Paulo: Edições, v. 70, 2011.
BRASIL. Ministério da Saúde. Política Nacional de Saúde do Trabalhador e da Trabalhadora. Portaria $\mathrm{n}^{\circ} \quad 1.823 ; \quad 2012$. Disponível em: $<$ http://ames.eng.br/uploads/arquivos/legislacao/Porta ria_MS_n.1283.pdf>. Acesso em: 2 Março 2017.

\section{Ministério da Saúde. Política Nacional de}

Humanização. Brasília, 256 p.: il. 2010.

Ministério da Saúde. Manual de estrutura física das unidades básicas de saúde: saúde da família. 2. ed. Brasil: Ministério da Saúde, 2008. (Série A. Normas e Manuais Técnicos)

DE ARAUJO FREITAS, Maílla Oliveira. O trabalhador da saúde na atenção básica: a experiência no cuidado à saúde. Revista de enfermagem UFPE on line-ISSN: 1981-8963, v. 9, n. 11, p. 9906-9912, $2015 . \quad$ Disponível em: $<$ https://periodicos.ufpe.br/revistas/revistaenfermage m/article/view/10785 >. Acesso em: 24 Março 2017.

DEJOURS, Christophe. Subjetividade, trabalho e ação. Revista Produção, São Paulo,v.14, n.3,p.27-34, $2004 . \quad$ Disponível em: $<$ http://www.scielo.br/scielo.php?script=sci_arttext\& pid=S0103-65132004000300004> Acesso em: 22 de Março de 2017.

HOGA, Luiza Akiko Komura. Cuidando do cuidador. In: Congresso Brasileiro de Enfermagem, 50. ABEn-Seçäo Bahia, 1998. p. 278-285. Disponível em: <http://sbmt.org.br/portal/wpcontent/uploads/2015/07/Anais-congresso-2014.pdf >. Acesso em 16 jul 2017.

LIMA, Thaís Jaqueline Vieira. Humanização na atenção à saúde do idoso. Saúde e Sociedade, p. 866877, 2010. Disponível em: <http://www.scielo.br/pdf/sausoc/v19n4/13.pdf >. Acesso em: 19 de Maio de 2017.

NEUMANN Vera Nilda. Qualidade de vida no trabalho: percepções da equipe de enfermagem na organização hospitalar. Belo Horizonte: 164f, 2007. Disponível em: <http://www.enf.ufmg.br/pos/defesas/359M.PDF>. Acesso em: 20 de Março de 2017.

TEIXEIRA, Sueli. A depressão no meio ambiente do trabalho e sua caracterização como doença do trabalho. Rev. Trib. Reg. Trab. $3^{\text {a }}$ Reg., Belo Horizonte, v.46, n.76, p.27-44, jul./dez.2007. Disponível em: 
<https://juslaboris.tst.jus.br/bitstream/handle/1939/73 993/2007_teixeira_sueli_depressao_meio.pdf?sequen
ce=1\&isAllowed=y>. Acesso em: 15 de Março de 2017. 